\title{
Universiteit
}

Leiden

The Netherlands

\section{Ehrenfest times for classically chaotic systems}

Beenakker, C.W.J.; Silvestrov, P.G.

\section{Citation}

Beenakker, C. W. J., \& Silvestrov, P. G. (2002). Ehrenfest times for classically chaotic systems. Retrieved from https://hdl.handle.net/1887/1219

Version: $\quad$ Not Applicable (or Unknown)

License: $\quad$ Leiden University Non-exclusive license

Downloaded from: https://hdl.handle.net/1887/1219

Note: To cite this publication please use the final published version (if applicable). 


\title{
Ehrenfest times for classically chaotic systems
}

\author{
P G Silvestiov ${ }^{2}$ and C W J Beenakker ${ }^{1}$ \\ ${ }^{1}$ Instltuut Lorentz Unversitelt Letden, PO Box 95062300 RA Leiden The Netherlands \\ ${ }^{2}$ Budker Insttute of Nucleal Physics, 630090 Novosibirsk, Russta \\ (Received 26 November 2001, published 7 March 2002)
}

\begin{abstract}
We describe the quantum-mechanical spreading of a Gaussian wave packet by means of the semiclassical WKB approximation of Berry and Balazs [J Phys A 2, 625 (1979)] We find that the time scale $\tau$ on which this approximation breaks down in a chaotic system is larger than the Ehrenfest times considered previously In one dimension $\tau=\frac{7}{6} \lambda^{-1} \ln (A / \hbar)$, with $\lambda$ the Lyapunov exponent and $A$ a typical classical action
\end{abstract}

According to Ehrenfest's theorem [1], the propagation of a quantum-mechanical wave packet is described for short times by classical equations of motion The time scale at which this conespondence between quantum and classical dynamıcs breaks down is called the Ehrenfest tıme If the classical dynamics is chaotic with Lyapunov exponent $\lambda$, then the Ehienfest time $\tau$ is of order $\lambda^{-1} \ln (A / \hbar)$ (with $A$ a typical classical action of the dynamical system) [2] There is actually more than a single Ehrenfest time, corresponding to different types of semiclassical approximations Although they differ only by a numerical coefficient, $\tau_{t}$ $=c_{1} \lambda^{-1} \ln (A / \hbar)$, the structure of the wave function changes qualitatively from one time scale to the next

Up to a time $\tau_{1}$, with $c_{1}=1 / 6$, the initial coherent state will retain its Gaussian form with vanishing errou in the limit $\hbar \rightarrow 0[3,4]$ For longer times up to $\tau_{2}$, with $c_{2}=1 / 2$, the uncertainty in the position and momentum of the particle remains small but the phase-space stuucture of the wave packet deviates strongly from a Gaussian For tımes greateı than $\tau_{2}$ the wave function no longer has the form of a wave packet (thıs is the "mıxıng regime" of Refs [5,6]), but up to a tıme $\tau_{3}$ it can still be described semiclassically by the tıme-dependent WKB approxımation of Berny and Balazs [7] As we will show in this paper, the WKB iepresentation implies $c_{3}=7 / 6$ for a single degree of freedom (with simple gener alızations for higher dimensions) This is larger than the value $c_{3}=2 / 3$ obtamed by Bouzouma and Robeıt [6] from a different semiclassical approximation

Let us start with the Gaussian one-dimensional wave packet

$$
\Psi(x)=\left(\frac{\alpha}{\pi \hbar}\right)^{1 / 4} \exp \left(\imath \frac{p_{0} x}{\hbar}+(l \beta-\alpha) \frac{\left(x-x_{0}\right)^{2}}{2 \hbar}\right)
$$

Inttally $\beta(t=0)=0$ and $\alpha(t=0)=p_{F} / L$, where $p_{F}$ and $L$ are the typical classical momentum and length The typical classical action is $A=p_{F} L$ The parameters $x_{0}(t), p_{0}(t)$ follow the classical trajectory for $\hbar \ll A$ We will measuie the momentum and cooldinate in units of $p_{F}$ and $L$, iespectively, so that $\alpha(0)=1$ and $A=1 \quad$ For chaotic dynamics with Lyapunov exponent $\lambda$ one has $\alpha(t) \propto \exp (-2 \lambda t)$, hence $\alpha$ $\ll 1$ for $t \gg 1 / \lambda$

To descibe the time evolution in phase space we consider the Wrgner function

$$
\begin{aligned}
W(x, p) & =\int \Psi\left(x+\frac{y}{2}\right) \Psi *\left(x-\frac{y}{2}\right) e^{-\imath p y / \hbar} \frac{d y}{2 \pi \hbar} \\
& =\frac{1}{\pi \hbar} \exp \left(-\frac{\alpha\left(x-x_{0}\right)^{2}}{\hbar}-\frac{\left[p-p_{0}-\beta\left(x-x_{0}\right)\right]^{2}}{\alpha \hbar}\right)
\end{aligned}
$$

The wave packet is centered at $x_{0}(t), p_{0}(t)$ and for $\alpha(t)$ $\ll 1$ becomes highly elongated and tilted with slope $\Delta p / \Delta x$ $\approx \beta \quad$ It has length $l_{\|}=\sqrt{\hbar\left(1+\beta^{2}\right) / \alpha}$ and width $l_{\perp}$ $=\sqrt{\hbar \alpha /\left(1+\beta^{2}\right)}$, so that the area in phase space is conserved exactly, $l_{\|} l_{\perp}=\hbar$ The Gaussian quantum wave packet satısfies the classical Liouville theorem

The Gausstan form (1) takes into account the elongation of the wave packet, but not the curvature that develops in tıme and results in a bending of the packet To describe the curvature we add an maginary cubic term in the exponent in Eq (1),

$$
\Psi(x)=\left(\frac{\alpha}{\pi \hbar}\right)^{1 / 4} \exp \left(\imath \frac{p_{0} x}{\hbar}+\frac{(\imath \beta-\alpha) x^{2}}{2 \hbar}+l \frac{\gamma x^{3}}{3 \hbar}\right)
$$

(For simplicity we have put $x_{0}=0$ ) The cubic term leads to an appieciable phase shift over a length $l_{\|} \simeq(\hbar / \alpha)^{1 / 2}$ when $(\gamma / \hbar)(\hbar / \alpha)^{3 / 2} \geq 1$, hence when $\alpha(t) \leqq \hbar^{1 / 3} \gamma^{2 / 3}$

For $\alpha \ll \hbar^{1 / 3} \gamma^{2 / 3}$ the Wigner function takes again a simple form, in terms of the Arry function $A_{1}$

$$
W(x, p)=\frac{\alpha^{1 / 2} \exp \left(-\alpha x^{2} / \hbar\right)}{\pi \hbar^{1 / 2}\left(\gamma \hbar^{2} / 4\right)^{1 / 3}} \mathrm{~A}_{1}\left(\frac{p_{0}+\beta x+\gamma x^{2}-p}{\left(\gamma \hbar^{2} / 4\right)^{1 / 3}}\right)
$$

One can check that $W(x, p) \rightarrow \delta(x) \delta\left(p-p_{0}\right)$ when $\hbar \rightarrow 0$ (at fixed $\alpha$ ), by means of the identity $\lim _{\varepsilon \rightarrow 0} \operatorname{Ar}(z / \varepsilon) / \varepsilon$ $=\sqrt{\pi} \delta(z)$ At finte $\hbar$ the wave packet is extended along the curved line $p=p_{0}+\beta x+\gamma x^{2}$ Since $p, p_{0}, x$ are of order unty, the two parameters $\beta$ and $\gamma$ are of order unity as well (in contiast to $\alpha$, which is $\ll 1$ ) The transverse width is of order

$$
l_{\perp} \approx \gamma^{1 / 3} \hbar^{2 / 3}\left(1+\beta^{2}\right)^{-1 / 2}
$$

The length of the packet remains at $l_{\|} \approx \sqrt{\hbar\left(1+\beta^{2}\right) / \alpha}$ Since now $l_{\|} l_{\perp} \gg \hbar$, the Liouville theorem no longer holds 
To obtain the Ehrenfest time, we parametrize time as

$$
t=\frac{c}{\lambda} \ln \frac{1}{\hbar}
$$

The classical limit for a chaotic system means $\hbar \rightarrow 0, t \rightarrow \infty$ at fixed $c$ Different coefficients $c$ follow from different sem1classical approximations If we use the Gaussian wave packet (1), without the cubic term to account for the curvature, then we need $\alpha(t) \gg \hbar^{1 / 3} \gamma^{2 / 3}$ Since $\alpha \propto e^{-2 \lambda t} \propto \hbar^{2 c}$ we need $c<1 / 6$ The upper limit of $c$ gives the first Ehıenfest tıme $\tau_{1}=\frac{1}{6} \lambda^{-1} \ln (1 / \hbar)$

The classical limit can be reached for longer times if we use the wave packet (3), including the cubic teim The d1mensions of the packet for $t>\tau_{1}$ scale with $\hbar$ as

$$
l_{\perp} \propto \hbar^{2 / 3}, \quad l_{\|} \propto \hbar^{1 / 2-c}
$$

For $c<1 / 2$ the length of the packet approaches zero in the classical limit This upper limit of $c$ gives the second Ehienfest time $\tau_{2}=\frac{1}{2} \lambda^{-1} \ln (1 / \hbar)$

For $t>\tau_{2}$ the length of the wave packet exceeds the size of the system and is no longer small compared to the iadius of curvature For these large tımes we may adopt the sem1classical WKB appioximation of Berry and Balazs [7] Consider a curve in phase space $p(x)$ and a phase-space distribution $\rho(p(x), x)$ Both $p$ and $\rho$ evolve in accordance with classical equations of motion For $t>\tau_{2}$ the function $p(x)$ is multivalued with an exponentially large numbel of branches $\sim \exp \left[\lambda\left(t-\tau_{2}\right)\right]$ The quantum wave function in this "mixing" regime has the form

$$
\Psi(x)=\sum_{k} f_{k}(x) \exp \left[l \sigma_{k}(x) / \hbar\right]
$$

The summation over $k$ accounts for the different bianches of the multivalued function $p(x)$ The two functions $f$ and $\sigma$ are related for $\hbar \rightarrow 0$ to $p$ and $\rho$ by the correspondence principle

$$
\frac{d \sigma}{d x}=p(x), \quad f=\sqrt{\rho(p, x)}
$$

An explicit description of the evolution of the wave function (8) for quantum maps can be found in Ref [8]

Near the point $x_{b}$ at which $p(x)$ bifuicates into two branches, one has $p=p_{b} \pm a \sqrt{x-x_{b}}, \quad \rho=b / \sqrt{x-x_{b}}$ The wave function there is

$$
\Psi=(\hbar / a)^{1 / 3} b^{1 / 2} \operatorname{Ar}\left[(a / \hbar)^{2 / 3}\left(x-x_{b}\right)\right] e^{l p_{b^{x}}}
$$

up to an overall phase The phase difference between the bifuication points can be deteimined from Eqs (8) and (9) Because the curve $p(x)$ is not closed, there is no analog of the Bohi-Sommerfeld quantization iule

The Wignel function conesponding to the wave function (8), being quadiatic in $\Psi$, contains both diagonal ( $W_{h k}$ $\propto\left|f_{k}\right|^{2}$ ) and oscillating nondiagonal $\left(W_{k m} \propto f_{k}^{\dagger} \hat{j}_{m}\right)$ contributions Far from bifurcations, the diagonal contibutions to the Wigner function read

$$
\begin{aligned}
W_{k k}(x, p) & =\int \exp \left(\frac{l y\left(\sigma^{\prime}-p\right)}{\hbar}+\frac{l y^{3} \sigma^{\prime \prime \prime}}{24 \hbar}\right) \frac{|f(x)|^{2} d y}{2 \pi \hbar} \\
& =\frac{2}{\sqrt{\pi}}\left(\frac{1}{\hbar^{2} \sigma^{\prime \prime \prime}}\right)^{1 / 3}|f(x)|^{2} \mathrm{Al}\left(\frac{2\left(\sigma^{\prime}-p\right)}{\left(\hbar^{2} \sigma^{\prime \prime \prime}\right)^{1 / 3}}\right)
\end{aligned}
$$

We have made a Taylor expansion of $\sigma(x \pm y / 2)$ and neglected the difference between $f(x \pm y / 2)$ and $f(x)$

If we parametize time as in Eq (6) we have for both $l_{\|}$ and $l_{\perp}$ the same scaling with $\hbar$ as in Eq (7) The range of validity of Eq (8) is limited by the condition that the diffe1ent branches should be distinguishable This 1equires that the different parts of the curve $p(x)$ in phase space should not get closer than $l_{\perp}$ Their spacing is of order $1 / l_{\|}$(assuming a uniform filling of phase space), hence

$$
l_{\|} l_{\perp} \ll 1 \Rightarrow \hbar^{7 / 6-c} \ll 1
$$

The upper limit of $7 / 6$ for $c$ leads to the thi1 Ehienfest time

$$
\tau_{3}=\frac{7}{6 \lambda} \ln \frac{1}{\hbar}
$$

The third derivative $\sigma^{\prime \prime \prime}$ in Eq (11) vanishes at the points of inflection of the curve $p(x)$ In ordel to find the Wigner function there, one should expand $\sigma(x \pm y / 2)$ up to terms of order $y^{5}$ This leads to a different scaling $l_{\perp} \propto \hbar^{4 / 5}$ of the width of the Wigner function near the inflection points Because these are isolated points, they will not contribute to the matrix elements of nonsingular operators (containing only smooth functions of $x$ and $p$ ) This different scaling should therefore not affect the Ehienfest time (13)

The nondiagonal contributions $W_{k m}$ to the Wigner function lead to the "ghost curves" discussed in Ref [9] (Ghost curves are tegions of large values of the Wigner function which do not coniespond to classical trajectories) The Wigner function near these curves is given by the same Al1y function as in Eq (11), but in addition acquires a strongly oscillating factor Due to these oscillations the nondiagonal teims do not contribute to the matı1x elements of nonsingular opesators (They may play a sole in the decoherence by the envilonment [10]) At $t \geqslant \tau_{3}$ the ghost cuives meige with the (multivalued) curve $p(x)$ and become indistmguishable

The time scale (13) for the bieakdown of the WKB approximation is gieater than the Ehrenfest time $\frac{2}{3} \lambda^{-1} \ln (1 / \hbar)$ in the mixed regime obtained in Ref [6] That shorter time scale may signal the breakdown of the senes expansion $\sigma_{k}(x) \rightarrow \Sigma_{j=0} \sigma_{k j}(x) \hbar^{j}$ Then Eq (9) would no longer hold, but for $t<\tau_{3}$ the repiesentation (8) with a renormalized function $\sigma_{h}(x)$ would still be valıd

So fal we have discussed a one-dimensional (1D) chaotic system, which in general can be represented by an area preserving map [8] A familiar example is the kicked rotator [11] For mesoscopic quantum dots, however, a more relevant model is the $d$-dimensional $(d=2,3)$ Schrodinger equation with a smooth potential $V(\vec{r})$ The Gaussian wave packet then takes the form 


$$
\Psi(\vec{r}) \propto \exp \left[\frac{l}{\hbar}\left(S\left(\vec{r}_{0}(t)\right)+\vec{p}_{0} \vec{x}+\frac{\zeta_{l n}}{2} x_{l} x_{n}\right)\right]
$$

Here $S$ is the action for the classical trajectory $\vec{r}_{0}(t)$ and we have defined $\vec{p}_{0}=m \vec{r}_{0}, \vec{x}=\vec{r}-\vec{r}_{0}, \zeta_{l n}=\beta_{l n}+\imath \alpha_{l n} \quad$ As before, we rescale the momentum and coordinate such that the typical classical action $A=1$ Initially, $\zeta_{l n} \simeq{ }_{l} \delta_{l n}$ Simila1 to the one dimensional case, $\alpha_{l n}$ defines the form of the packet in coordinate space and $\beta_{l n}=\Delta p_{l} / \Delta x_{n}$ give the angles in phase space Substituting the wave function (14) into the Schrodinger equation one finds Newton's equation of motion for $\vec{r}_{0}$ The spreading of the wave packet in phase space is described by

$$
-\zeta_{l n}=\frac{1}{m} \zeta_{l k} \zeta_{k n}+\left.\frac{\partial^{2} V}{\partial r_{l} \partial r_{n}}\right|_{i_{i=i_{0}}}
$$

This is the equation descilbing the spreading in phase space of a small Gaussian bunch of classical paiticles

The Wigner function conesponding to the wave function (14) has the Gaussian form $W \propto \exp \left(-Q_{l} M_{l n} Q_{n} / \hbar\right)$, where $\vec{Q}=\left(\vec{r}-\vec{r}_{0}, \vec{p}-\vec{p}_{0}\right)$ is a vectol in $2 d$-dimensional phase space The $d$ Lyapunov exponents $\lambda_{t}(l=1,2, \quad, d)$ govern the large-tume behavior of the e1genvalues $m_{t}=1 / m_{2 d-l+1}$ $\propto \exp \left(2 \lambda_{l} t\right)$ of the 1eal symmetric mat11x $M$ Because of eneigy conservation one Lyapunov exponent vanishes We order the $\lambda$ 's from large to small, so that $\lambda_{1}$ is the largest and $\lambda_{d}=0$

The wave packet 1emains Gaussian (preserving the volume $\propto \hbar^{d}$ in phase space) untıl the cuivature starts to play a role (via a cubic teim in the action) The corresponding Ehienfest time $\tau_{1}=\frac{1}{6} \lambda_{1}^{-1} \ln (1 / \hbar)$ is the same as in $1 D$, only now it is defined through the la1gest Lyapunov exponent $\lambda_{1}$ The second Ehrenfest time, when the length of the packet exceeds the size of the system, also has the same form $\tau_{2}$ $=\frac{1}{2} \lambda_{1}^{-1} \ln (1 / \hbar)$

The third time $\tau_{3}$ is different for $d=2,3$ from the $1 \mathrm{D}$ case Instead of $\mathrm{Eq}(7)$, one now has

$$
l_{\perp}^{(l)} \propto \hbar^{2 / 2}, \quad l_{\|}^{(l)} \propto \hbar^{1 / 2} e^{\lambda, t}, \quad l=1,2, \quad, d-1
$$

The longitudinal dimensions $l_{\|}^{(1)}$ correspond to eigenvalues $m_{l}$ with $1 \leqslant l \leqslant d-1$, and the tiansveise dimensions $l_{\perp}^{(l)}$ to $m_{l}$ with $d+2 \leqslant l \leqslant 2 d$ The two unit eigenvalues $m_{d}=m_{d+1}$ $=1$ contribute another factor $\sqrt{\hbar}$ each to the total volume $\mathcal{V}$ in phase space covered by the wave packet

$$
\nu=\hbar \prod_{l=1}^{d-1} l_{\perp}^{(l)} l_{l}^{(l)} \propto \hbar^{7 d / 6-1 / 6} e^{\lambda_{\text {tot }}{ }^{l},} \quad \lambda_{\text {tot }}=\sum_{i=1}^{d-1} \lambda_{l}
$$

The avalable area $\mathcal{V}_{\max } 1 \mathrm{~s}$ restricted to a shell of constant energy with thickness $\sqrt{\hbar}$, hence $\mathcal{V}_{\operatorname{mix}} \propto \sqrt{\hbar}$ We requile $\mathcal{V}$ $\leqslant V_{\max }$ for the semiclassical appioximation, which leads to the Ehrenfest time

$$
\tau_{3}=\frac{7 d-4}{6 \lambda_{\text {tot }}} \ln \frac{A}{\hbar}, d \geqslant 2
$$

In conclusion, we exammed different time scales $\tau_{l}$ $=c_{l} \lambda^{-1} \ln (1 / \hbar)$ for the bieakdown of different types of semiclassical appioximations These Ehrenfest times differ only by a numerical coefficient $c_{l}$, which may seem insignificant However, this difference is actually a signal of a different power law scaling with $\hbar$ of the volume $\mathcal{V}$ in phase space covered by the wave packet For short times Liouville's theorem dictates $\mathcal{V} \propto \hbar$ For long times [parameterized as $t=(c / \lambda) \ln (1 / \hbar)]$ the WKB approximation gives $\mathcal{V}$ $\propto \hbar^{7 / 6-c}$ for a one-dımensional quantum map (such as the kicked 1otatol) and $\mathcal{V} \propto \hbar^{7 d / 6-1 / 6-c}$ for a $d$-dimensional conselvative system These different power laws reflect the fundamental change in the stiucture of the wave function with increasing time and should, therefore, have observable consequences Two possible applications are the Loschmidt echo [12] and the quantum shot nosse [13], where the Ehrenfest time plays a key role

This work was supported by the Dutch Science Foundation NWO/FOM and by the National Science Foundation unde1 Grant No PHY99-07949
[1] P Ehienfest, Z Phys 45, 455 (1927)

[2] G P Berman and G M Zaslavsky, Physica A 91, 450 (1978), G M Zaslavsky, Phys Rep 80, 157 (1981)

[3] M Combescure and D Robert, Asymptotic Anal 14, 377 (1997)

[4] G A Hagedorn and A Joye, Ann Henrı Poincaré 1, 837 (2000)

[5] F Bonech1 and S De Bievre, Commun Math Phys 211, 659 (2000)

[6] A Bouzouina and D Robert, Duke Math J (to be published)

[7] M V Berry and N L Balazs J Phys A 12, 625 (1979)

[8] M V Berry, N L Balazs, M Tabor, and A Voros, Ann Phys
(N Y) 122, $26(1979)$

[9] M A Alonso and GW Forbes, J Opt Soc Am A 17, 2288 (2000)

[10] W H Zurek, Nature (London) 412, 712 (2001)

[11] G Casat1, B V Chirıkov, FM Izrallev, and J Ford, Lect Notes Phys 93, 334 (1979)

[12] R A Jalabert and HM Pastawsk1, Phys Rev Lett 86, 2490 (2001), Ph Jacquod, PG Silvestrov, and CW J Beenakker, Phys Rev E 64, 055203(R) (2001)

[13] O Agam, I Aleıner, and A Larkın, Phys Rev Lett 85, 3153 (2000), I L Aleıner and A I Larkın, Phys Rev B 54, 14423 (1996) 